\title{
ASYMPTOTIC BEHAVIOUR OF CERTAIN SETS OF PRIME IDEALS
}

\author{
ALAN K. KINGSBURY AND RODNEY Y. SHARP \\ (Communicated by Wolmer V. Vasconcelos)
}

\begin{abstract}
Let $I_{1}, \ldots, I_{g}$ be ideals of the commutative ring $R$, let $M$ be a Noetherian $R$-module and let $N$ be a submodule of $M$; also let $A$ be an Artinian $R$-module and let $B$ be a submodule of $A$. It is shown that, whenever $\left(a_{m}(1), \ldots, a_{m}(g)\right)_{m \in \mathbb{N}}$ is a sequence of $g$-tuples of non-negative integers which is non-decreasing in the sense that $a_{i}(j) \leq a_{i+1}(j)$ for all $j=1, \ldots, g$ and all $i \in \mathbb{N}$, then $\operatorname{Ass}_{R}\left(M / I_{1}^{a_{n}(1)} \ldots I_{g}^{a_{n}(g)} N\right)$ is independent of $n$ for all large $n$, and also $\operatorname{Att}_{R}\left(B:_{A} I_{1}^{a_{n}(1)} \ldots I_{g}^{a_{n}(g)}\right)$ is independent of $n$ for all large $n$. These results are proved without any regularity conditions on the ideals $I_{1}, \ldots, I_{g}$, and so (a special case of) the first answers in the affirmative a question raised by S. McAdam.
\end{abstract}

\section{INTRODUCTION}

Let $R$ be a commutative ring (with identity) and $I$ be an ideal of $R$. In [Ra] L. J. Ratliff, Jr., conjectured about the asymptotic behaviour of $\operatorname{Ass}_{R}\left(R / I^{n}\right)$ when $R$ is a Noetherian domain. Subsequently, M. Brodmann [B] showed that if $R$ is Noetherian and $M$ is a finitely generated $R$-module, then $\operatorname{Ass}_{R}\left(M / I^{n} M\right)$ is ultimately constant for large $n \in \mathbb{N}$. (We use $\mathbb{N}_{0}$ (respectively $\mathbb{N}$ ) to denote the set of non-negative (respectively positive) integers.) It is a very easy consequence of Brodmann's result that the same conclusion holds if we relax the hypotheses and assume only that $R$ is a commutative ring and $M$ is a Noetherian $R$-module. In [S1, Theorem 3.1(iii)] the second author proved that if $A$ is an Artinian $R$-module, then $\operatorname{Att}_{R}\left(0:_{A} I^{n}\right)$ is ultimately constant for large $n$; in a sense, this can be regarded as a dual of Brodmann's result. More recently in [Ru, Theorem 5.5] D. E. Rush further expanded upon this by showing that if $N$ is an $R$-submodule of the Noetherian $R$-module $M$, then $\operatorname{Ass}_{R}\left(M / I^{n} N\right)$ is ultimately constant for large $n \in \mathbb{N}$, and if $B$ is an $R$-submodule of the Artinian $R$-module $A$, then $\operatorname{Att}_{R}\left(B:_{A} I^{n}\right)$ is ultimately constant for large $n \in \mathbb{N}$.

On a different course of investigation, D. Katz, S. McAdam and L. J. Ratliff, Jr., have shown in [K-M-R, Corollary 1.8(c)] that if $R$ is Noetherian, $I_{1}, \ldots, I_{g}$ are regular ideals of $R$, and $\left(a_{m}(1), \ldots, a_{m}(g)\right)_{m \in \mathbb{N}}$ is a sequence of $g$-tuples of non-negative integers which is non-decreasing in the sense that $a_{i}(j) \leq a_{i+1}(j)$ for

Received by the editors December 13, 1994.

1991 Mathematics Subject Classification. Primary 13E05, 13E10.

Key words and phrases. Associated prime ideal, attached prime ideal, Noetherian module, Artinian module, asymptotic prime divisors. 
all $j=1, \ldots, g$ and all $i \in \mathbb{N}$, then $\operatorname{Ass}_{R}\left(R / I_{1}^{a_{n}(1)} \ldots I_{g}^{a_{n}(g)}\right)$ is independent of $n$ for all large $n$. Further in [Mc, 13.5(i)] S. McAdam put forward the question: "Can we drop the assumption that $I_{1}, \ldots, I_{g}$ are regular"; one of the main results of this paper is an affirmative answer to this question.

This paper is concerned with what might be considered a natural generalisation of the above-mentioned result of Katz, McAdam and Ratliff. Let $I_{1}, \ldots, I_{g}$ be ideals of $R$ and let $\left(a_{m}(1), \ldots, a_{m}(g)\right)_{m \in \mathbb{N}}$ be a sequence of $g$-tuples of non-negative integers which is non-decreasing. We shall show that if $N$ is a submodule of the Noetherian $R$-module $M$ and $B$ is a submodule of the Artinian $R$-module $A$, then $\operatorname{Ass}_{R}\left(M / I_{1}^{a_{n}(1)} \ldots I_{g}^{a_{n}(g)} N\right)$ and $\operatorname{Att}_{R}\left(B:_{A} I_{1}^{a_{n}(1)} \ldots I_{g}^{a_{n}(g)}\right)$ are both ultimately constant for large $n$. We shall use techniques similar to those employed by Katz, McAdam and Ratliff to establish the first result when $R$ is Noetherian, $M$ is finitely generated and the ideals satisfy some regularity conditions. Then we shall extend the result to the general case by use of the Artin-Rees Lemma. In Section 2, we shall use Matlis duality and techniques investigated by the second author in [S3], which allow an Artinian module over a commutative ring to be considered as a faithful Artinian module over a complete semi-local Noetherian ring, to prove the second result. (Throughout this paper whenever we describe a semi-local Noetherian ring as "complete", we shall always mean it is complete with respect to the topology defined by its Jacobson radical.)

\section{Associated PRIMe ideals}

1.1 Notation. Throughout this paper $R$ will denote a commutative ring (with identity). For a fixed positive integer $j$, we shall denote by $\mathbb{N}_{0}^{j}$ the set of all $j$-tuples of non-negative integers. Throughout, let $g$ be a fixed positive integer, and let $I_{1}, \ldots, I_{g}$ be ideals of $R$; and for $n=\left(n_{1}, \ldots, n_{g}\right) \in \mathbb{N}_{0}^{g}$ we shall denote $I_{1}^{n_{1}} \ldots I_{g}^{n_{g}}$ by $I^{n}$. Let $j \in \mathbb{N}$. For $n \in \mathbb{N}_{0}^{j}$ we define $n(i)$ to be the $i$ th component of $n$ $(1 \leq i \leq j)$. Thus for $n, m \in \mathbb{N}_{0}^{j}$, by $n \leq m$ (respectively $n<m$ ) we mean $n(i) \leq m(i)$ (respectively $n(i)<m(i))$ for all $1 \leq i \leq j$. Addition, and multiplication by elements of $\mathbb{N}_{0}$, are defined on $\mathbb{N}_{0}^{j}$ in the obvious ways. We denote by $\mathbb{Z}^{j}$ the set of all $j$-tuples of integers, defining operations involving elements of $\mathbb{Z}^{j}$ in a similar fashion as for $\mathbb{N}_{0}^{j}$, with the convention that if $z(i)<0$ for some $1 \leq i \leq j$, then we take $I_{i}^{z(i)}$ to be $R$.

1.2 Lemma. Let $R$ be a Noetherian ring, let $M$ be a finitely generated $R$-module and let $N$ be a submodule of $M$. Then the set $\bigcup_{n \in \mathbb{N}_{0}^{g}} \operatorname{Ass}_{R}\left(M / I^{n} N\right)$ is finite.

Proof. For $z=\left(z_{1}, \ldots, z_{g}\right) \in \mathbb{Z}^{g}$ we shall denote $t_{1}^{z_{1}} \ldots t_{g}^{z_{g}}$ by $t^{z}$. Let $\mathfrak{R}$ denote $R\left[I_{1} t_{1}, \ldots, I_{g} t_{g}, t_{1}^{-1}, \ldots, t_{g}^{-1}\right]$, the extended Rees ring of $I_{1}, \ldots, I_{g}$. We denote $R\left[t_{1}, \ldots, t_{g}, t_{1}^{-1}, \ldots, t_{g}^{-1}\right] \otimes_{R} M$ by $M\left[t_{1}, \ldots, t_{g}, t_{1}^{-1}, \ldots, t_{g}^{-1}\right]$ and denote its element $t^{z} \otimes m$ (for $z \in \mathbb{Z}^{g}$ and $m \in M$ ) by $m t^{z}$. Let $\mathfrak{M}$ denote $\bigoplus_{z \in \mathbb{Z}^{g}} I^{z} M t^{z}$, a $\mathbb{Z}^{g}$-graded $\mathfrak{R}$-submodule of $M\left[t_{1}, \ldots, t_{g}, t_{1}^{-1}, \ldots, t_{g}^{-1}\right]$. Similarly let $\mathfrak{N}$ denote $\bigoplus_{z \in \mathbb{Z}^{g}} I^{z} N t^{z}$, a $\mathbb{Z}^{g}$-graded $\mathfrak{R}$-submodule of $\mathfrak{M}$. Let $n \in \mathbb{N}_{0}^{g}$. It is clear that $t^{-n} \mathfrak{N}$ is a homogeneous $\mathfrak{R}$-submodule of $\mathfrak{M}$ with the $(0, \ldots, 0)$-th component $I^{n} N$. Hence

$$
\operatorname{Ass}_{R}\left(M / I^{n} N\right) \subseteq\left\{\mathfrak{P} \cap R: \mathfrak{P} \in \operatorname{Ass}_{\mathfrak{R}}\left(\mathfrak{M} / t^{-n} \mathfrak{N}\right)\right\}
$$


There are three possibilities for $n$ : we have (i) $n=0$, or (ii) $t^{-n}=t_{i}^{-1}$ for some $1 \leq i \leq g$, or (iii) there exists $k \in \mathbb{N}$ with $1 \leq k \leq g$ such that $n(k) \neq 0$ and $t^{-n}=t_{k}^{-1} t^{-n^{\prime}}$ for some $0 \neq n^{\prime} \in \mathbb{N}_{0}^{g}$. Then since $t_{k}^{-1}$ is a non-zerodivisor on $\mathfrak{M}$, there is an exact sequence

$$
0 \longrightarrow \mathfrak{M} / t^{-n^{\prime}} \mathfrak{N} \longrightarrow \mathfrak{M} / t_{k}^{-1} t^{-n^{\prime}} \mathfrak{N} \longrightarrow \mathfrak{M} / t_{k}^{-1} \mathfrak{M} \longrightarrow 0
$$

and so

$$
\operatorname{Ass}_{\mathfrak{R}}\left(\mathfrak{M} / t^{-n} \mathfrak{N}\right) \subseteq \operatorname{Ass}_{\mathfrak{R}}\left(\mathfrak{M} / t_{k}^{-1} \mathfrak{M}\right) \cup \operatorname{Ass}_{\mathfrak{R}}\left(\mathfrak{M} / t^{-n^{\prime}} \mathfrak{N}\right)
$$

Repetition of this argument shows that, in cases (ii) or (iii),

$$
\operatorname{Ass}_{\mathfrak{R}}\left(\mathfrak{M} / t^{-n} \mathfrak{N}\right) \subseteq \bigcup_{1 \leq k \leq g}\left(\operatorname{Ass}_{\mathfrak{R}}\left(\mathfrak{M} / t_{k}^{-1} \mathfrak{M}\right) \cup \operatorname{Ass}_{\mathfrak{R}}\left(\mathfrak{M} / t_{k}^{-1} \mathfrak{N}\right)\right)
$$

Hence

$$
\begin{gathered}
\bigcup_{n \in \mathbb{N}_{0}^{g}} \operatorname{Ass}_{R}\left(M / I^{n} N\right) \subseteq\left\{\mathfrak{P} \cap R: \mathfrak{P} \in \bigcup_{n \in \mathbb{N}_{0}^{g}} \operatorname{Ass}_{\mathfrak{R}}\left(\mathfrak{M} / t^{-n} \mathfrak{N}\right)\right\} \\
\subseteq\left\{\mathfrak{P} \cap R: \mathfrak{P} \in\left(\bigcup_{1 \leq k \leq g}\left(\operatorname{Ass}_{\mathfrak{R}}\left(\mathfrak{M} / t_{k}^{-1} \mathfrak{M}\right) \cup \operatorname{Ass}_{\mathfrak{R}}\left(\mathfrak{M} / t_{k}^{-1} \mathfrak{N}\right)\right) \bigcup \operatorname{Ass}_{\mathfrak{R}}(\mathfrak{M} / \mathfrak{N})\right)\right\},
\end{gathered}
$$

which clearly is a finite set.

1.3 Lemma. Let $R$ be a Noetherian ring and let $M$ be a finitely generated $R$ module. Let $h$ be a fixed positive integer and let $J_{1}, \ldots, J_{h}$ be ideals of $R$. Let $J^{n}$, for $n \in \mathbb{N}_{0}^{h}$, be defined in a similar fashion to $I^{n^{\prime}}$, for $n^{\prime} \in \mathbb{N}_{0}^{g}$, in 1.1. Also for fixed $i$, we define $K^{s}$ for $s \in \mathbb{N}_{0}^{h-1}$ to be $J_{1}^{s(1)} \ldots J_{i-1}^{s(i-1)} J_{i+1}^{s(i)} \ldots J_{h}^{s(h-1)}$.

(i) For each $i$, with $1 \leq i \leq h$, if $J_{i}$ is $M$-regular, then there exists $c_{i} \in \mathbb{N}_{0}$ such that $\left(J_{i}^{d_{i}+e} K^{s} M:_{M} J_{i}^{e}\right)=J_{i}^{d_{i}} K^{s} M$ for all $s \in \mathbb{N}_{0}^{h-1}, d_{i}, e \in \mathbb{N}_{0}$ with $d_{i}>c_{i}$.

(ii) Let $H$ be an ideal of $R$ and suppose that $J_{1}, \ldots, J_{h}$ are $M$-regular. Then there exists $t \in \mathbb{N}_{0}^{h}$ such that $\left(J^{n+m} H M:_{M} J^{m}\right)=J^{n} H M$ for all $n, m \in \mathbb{N}_{0}^{h}$ with $n>t$.

Proof. (i) We may clearly assume that $M \neq 0$ (and $J_{i} \neq 0$ ). Let

$$
M=m_{1} R+\ldots+m_{r} R \text {. }
$$

Fix $i \in \mathbb{N}$ with $1 \leq i \leq h$, and assume that $J_{i}$ is $M$-regular. Then there exist $M$ regular elements $a_{1}, \ldots, a_{t} \in R$ such that $J_{i}=a_{1} R+\ldots+a_{t} R$. For $j=1, \ldots, t$, let $S_{j}=\left\{a_{j}^{r}: r \in \mathbb{N}_{0}\right\}$, and let $N, L$ be the $R$-submodules of $S_{1}^{-1} M \oplus \ldots \oplus S_{t}^{-1} M$ given by $N=\{(m / 1, \ldots, m / 1): m \in M\}$ and $L=\left\{\left(m_{1}^{\prime} / a_{1}, \ldots, m_{t}^{\prime} / a_{t}\right): m_{1}^{\prime}, \ldots, m_{t}^{\prime} \in\right.$ $M\}$. Clearly $N \subseteq L$, and $L$ is finitely generated. Define $\phi: M \rightarrow N$ by $\phi(m)=$ $(m / 1, \ldots, m / 1)$ for all $m \in M$, so that $\phi$ is an isomorphism. Now

$$
\begin{aligned}
& J^{n} L \cap N=\left\{(m / 1, \ldots, m / 1): m \in M, a_{j} m / a_{j}=l_{j} / a_{j} \text { with } l_{j} \in J^{n} M\right. \text { for all } \\
&j=1, \ldots, t\}
\end{aligned}
$$


for all $n \in \mathbb{N}_{0}^{h}$. Thus by [K-M-R, Proposition 1.4], there exists $c_{i} \in \mathbb{N}_{0}$ such that

$$
\begin{aligned}
\phi\left(\left(J_{i}^{d_{i}+f} K^{s} M:_{M} J_{i}\right)\right) & =J_{i}^{d_{i}+f} K^{s} L \cap N \\
& =J_{i}^{f}\left(J_{i}^{d_{i}} K^{s} L \cap N\right) \\
& =\phi\left(J_{i}^{f}\left(J_{i}^{d_{i}} K^{s} M:_{M} J_{i}\right)\right) \\
& \subseteq \phi\left(J_{i}^{d_{i}+f-1} K^{s} M\right)
\end{aligned}
$$

for all $s \in \mathbb{N}_{0}^{h-1}, d_{i}, f \in \mathbb{N}_{0}$ with $d_{i}>c_{i}$ and $f \geq 1$. Let $e \in \mathbb{N}_{0}$ with $e \geq 1$ and $x \in\left(J_{i}^{d_{i}+e} K^{s} M: J_{i}^{e}\right)$ for some $d_{i}>c_{i}$. Then $x J_{i}^{e} \subseteq J_{i}^{d_{i}+e} K^{s} M$, so $x J_{i}^{e-1} \subseteq$ $\left(J_{i}^{d_{i}+e} K^{s} M:_{M} J_{i}\right) \subseteq J_{i}^{d_{i}+e-1} K^{s} M$, and continuing inductively in this manner we get $x \in J_{i}^{d_{i}} K^{s} M$. Hence $\left(J_{i}^{d_{i}+e} K^{s} M:_{M} J_{i}^{e}\right)=J_{i}^{d_{i}} K^{s} M$ for all $s \in \mathbb{N}_{0}^{h-1}$, $d_{i}, e \in \mathbb{N}_{0}$ with $d_{i}>c_{i}$, as required.

(ii) This follows easily by repeated application of the first part.

1.4 Theorem. Let $R$ be a Noetherian ring and let $M$ be a finitely generated $R$ module.

(i) Let $\left(a_{m}\right)_{m \in \mathbb{N}}$ be a sequence in $\mathbb{N}_{0}^{g}$ with $a_{i} \leq a_{i+1}$ for all $i \in \mathbb{N}$. Assume that $I_{i}$ is $M$-regular for each $i$ with $1 \leq i \leq g$ such that $\left\{a_{j}(i): j \in \mathbb{N}\right\}$ is infinite. Then $\operatorname{Ass}_{R}\left(M / I^{a_{m}} M\right)$ is ultimately constant for large $m$.

(ii) Furthermore, if $I_{i}$ is $M$-regular for all $i=1, \ldots, g$, then there exists $d \in \mathbb{N}^{g}$ such that $\operatorname{Ass}_{R}\left(M / I^{n} M\right)$ is independent of $n$ for all $n \geq d$.

Proof. (i) If $\left\{a_{j}(i): j \in \mathbb{N}\right\}$ is finite for all $i=1, \ldots, g$, then the result is clear. Therefore we suppose this is not the case and we may reorder the ideals so that for some $q \in \mathbb{N}$ with $1 \leq q \leq g,\left\{a_{j}(i): j \in \mathbb{N}\right\}$ is infinite for $1 \leq i \leq q$, and finite for $q<i \leq g$. Define $b_{m}:=\left(a_{m}(1), \ldots, a_{m}(q)\right) \in \mathbb{N}_{0}^{q}$ and denote $I_{1}^{b_{m}}(1) \ldots I_{q}^{b_{m}(q)}$ by $J^{b_{m}}$ for all $m \in \mathbb{N}$. Then there exists an ideal $H$ of $R$ such that $I^{a_{m}}=J^{b_{m}} H$ for all sufficiently large $m$, and it suffices to show that $\operatorname{Ass}_{R}\left(M / J^{b_{m}} H M\right)$ is ultimately constant for large $m$.

Now by 1.3 , for all sufficiently large $m, J^{b_{m}} H M=\left(J^{b_{m+1}} H M:_{M} J^{b_{m+1}-b_{m}}\right)$. Let $P \in \operatorname{Ass}_{R}\left(M / J^{b_{m}} H M\right)$ for such an $m$, so that $P=\left(0:_{R} x+J^{b_{m}} H M\right)$ for some $x \in M \backslash J^{b_{m}} H M$. It follows that $r \in\left(0:_{R} x+J^{b_{m}} H M\right)$ if and only if $r \in$ $\left(0:_{R}\left(J^{b_{m+1}-b_{m}} x+J^{b_{m+1}} H M\right) / J^{b_{m+1}} H M\right)$, and so $P \in \operatorname{Ass}_{R}\left(M / J^{b_{m+1}} H M\right)$. Now by 1.2 ,

$$
\bigcup_{m \in \mathbb{N}} \operatorname{Ass}_{R}\left(M / J^{b_{m}} H M\right) \subseteq \bigcup_{n \in \mathbb{N}_{0}^{q}} \operatorname{Ass}_{R}\left(M / I_{1}^{n(1)} \ldots I_{q}^{n(q)} H M\right)
$$

is finite, and hence it follows that $\operatorname{Ass}_{R}\left(M / I^{a_{m}} M\right)$ is ultimately constant for large $m$.

(ii) By 1.3(ii), there exists $k \in \mathbb{N}_{0}^{g}$ such that $I^{n} M=\left(I^{n+m} M:_{M} I^{m}\right)$ for all $n, m \in \mathbb{N}_{0}^{g}$ with $n>k$. Let $s^{\prime} \geq t^{\prime}>k$ and let $P \in \operatorname{Ass}_{R}\left(M / I^{t^{\prime}} M\right)$, so that $P=\left(0:_{R} x+I^{t^{\prime}} M\right)$ for some $x \in M \backslash I^{t^{\prime}} M$. It follows that $r \in\left(0:_{R} x+I^{t^{\prime}} M\right)$ if and only if $r \in\left(0:_{R}\left(I^{s^{\prime}-t^{\prime}} x+I^{s^{\prime}} M\right) / I^{s^{\prime}} M\right)$, and so $P \in \operatorname{Ass}_{R}\left(M / I^{s^{\prime}} M\right)$. 
For each $j \in \mathbb{N}$, let $n_{j}=(j, \ldots, j) \in \mathbb{N}^{g}$. Then, by part (i), there exists $l \in \mathbb{N}$ such that

$$
\operatorname{Ass}_{R}\left(M / I^{n_{l}} M\right)=\operatorname{Ass}_{R}\left(M / I^{n_{l+i}} M\right) \quad \text { for all } i \in \mathbb{N} .
$$

Let $c=\max \{l, k(1)+1, \ldots, k(g)+1\}$ and define $d:=n_{c}$. Let $s \in \mathbb{N}^{g}$ with $s \geq d$. Then there exists $r \in \mathbb{N}$ such that $r d \geq s$, and hence, since $d>k$,

$$
\operatorname{Ass}_{R}\left(M / I^{d} M\right) \subseteq \operatorname{Ass}_{R}\left(M / I^{s} M\right) \subseteq \operatorname{Ass}_{R}\left(M / I^{r d} M\right)=\operatorname{Ass}_{R}\left(M / I^{d} M\right) .
$$

In the next theorem, there is no 'regularity' assumption on the ideals $I_{1}, \ldots, I_{g}$. Thus the particular case of the theorem in which $M=R$ answers in the affirmative a question raised by McAdam in [Mc, 13.5(i)].

1.5 Theorem. Let $R$ be a Noetherian ring, let $M$ be a finitely generated $R$-module and let $N$ be a submodule of $M$.

(i) Let $\left(a_{m}\right)_{m \in \mathbb{N}}$ be a sequence in $\mathbb{N}_{0}^{g}$ with $a_{i} \leq a_{i+1}$ for all $i \in \mathbb{N}$. Then $\operatorname{Ass}_{R}\left(M / I^{a_{m}} N\right)$ is ultimately constant for large $m$.

(ii) Furthermore, there exists $d \in \mathbb{N}^{g}$ such that $\operatorname{Ass}_{R}\left(M / I^{n} N\right)$ is independent of $n$ for all $n \geq d$.

Proof. (i) The result is obvious if $\left\{a_{j}(i): j \in \mathbb{N}\right\}$ is finite for all $1 \leq i \leq g$. Hence we shall assume this is not the case and we may reorder the ideals so that there exists $q \in \mathbb{N}$ with $1 \leq q \leq g$ such that $\left\{a_{j}(i): j \in \mathbb{N}\right\}$ is infinite for $1 \leq i \leq q$ and finite for $q<i \leq g$. Define $b_{m}:=\left(a_{m}(1), \ldots, a_{m}(q)\right) \in \mathbb{N}_{0}^{q}$ and denote $I_{1}^{b_{m}(1)} \ldots I_{q}^{b_{m}(q)}$ by $J^{b_{m}}$ for all $m \in \mathbb{N}$. Then there exists an ideal $K$ of $R$ such that $I^{a_{m}}=J^{b_{m}} K$ for all $m \geq l$, say, and it suffices to show that $\operatorname{Ass}_{R}\left(M / J^{b_{m}} K N\right)$ is ultimately constant for large $m$.

By applying the Artin-Rees Lemma to $\left(0:_{N} I_{i}\right) \subseteq N$, we see that, for each $i=1, \ldots, q$, there exists $h_{i} \in \mathbb{N}_{0}$ such that

$$
\left(0:_{I_{i}^{h_{i}+1} N} I_{i}\right)=I_{i}^{h_{i}+1} N \cap\left(0:_{N} I_{i}\right)=I_{i}\left(I_{i}^{h_{i}} N \cap\left(0:_{N} I_{i}\right)\right)=0 .
$$

Let $L=I_{1}^{h_{1}+1} \ldots I_{q}^{h_{q}+1} K$. Then, for each $i=1, \ldots, q$, we have $\left(0:_{L N} I_{i}\right)=0$, so that $I_{i}$ is $L N$-regular.

Now there exists $l^{\prime} \in \mathbb{N}$ with $l^{\prime} \geq l$ such that $b_{m}(i) \geq h_{i}+1$ for all $m \geq l^{\prime}$ and $1 \leq i \leq q$. Then for $m \geq l^{\prime}$ we define

$$
c_{m}:=\left(b_{m}(1)-h_{1}-1, \ldots, b_{m}(q)-h_{q}-1\right) \in \mathbb{N}_{0}^{q},
$$

and $J^{c_{m}}$ in a way similar to $J^{b_{m}}$. Then $I^{a_{m}}=J^{c_{m}} L$ for all $m \geq l^{\prime}$. Now for all $m \geq l^{\prime}$, there is an exact sequence

$$
0 \longrightarrow J^{c_{m}} L N / J^{c_{m+1}} L N \longrightarrow M / I^{a_{m+1}} N \longrightarrow M / I^{a_{m}} N \longrightarrow 0
$$

and so

$$
\operatorname{Ass}_{R}\left(M / I^{a_{m+1}} N\right) \subseteq \operatorname{Ass}_{R}\left(J^{c_{m}} L N / J^{c_{m+1}} L N\right) \cup \operatorname{Ass}_{R}\left(M / I^{a_{m}} N\right) .
$$


By 1.4(i), we know that $\operatorname{Ass}_{R}\left((L N) / J^{c_{m+1}}(L N)\right)$ is ultimately constant for large $m$. Thus for all sufficiently large $m$,

$$
\begin{aligned}
\operatorname{Ass}_{R}\left(J^{c_{m}} L N / J^{c_{m+1}} L N\right) & \subseteq \operatorname{Ass}_{R}\left(L N / J^{c_{m+1}} L N\right)=\operatorname{Ass}_{R}\left(L N / J^{c_{m}} L N\right) \\
& \subseteq \operatorname{Ass}_{R}\left(M / I^{a_{m}} N\right),
\end{aligned}
$$

so that, by $(*)$,

$$
\operatorname{Ass}_{R}\left(M / I^{a_{m+1}} N\right) \subseteq \operatorname{Ass}_{R}\left(M / I^{a_{m}} N\right) .
$$

Hence $\operatorname{Ass}_{R}\left(M / I^{a_{m}} N\right)$ is ultimately constant for large $m$.

(ii) For each $j \in \mathbb{N}$, let $n_{j}=(j, \ldots, j) \in \mathbb{N}^{g}$. Then by part (i), there exists $k \in \mathbb{N}$ such that

$$
\operatorname{Ass}_{R}\left(M / I^{n_{k}} N\right)=\operatorname{Ass}_{R}\left(M / I^{n_{k+i}} N\right) \quad \text { for all } i \in \mathbb{N} .
$$

As in the proof of part (i), we can apply the Artin-Rees Lemma to $\left(0:_{N} I_{i}\right) \subseteq N$ to see that, for each $i=1, \ldots, g$, there exists $x_{i} \in \mathbb{N}_{0}$ such that $\left(0:_{i_{i}^{x_{i}+1}{ }_{N}} I_{i}\right)=0$. Let $y=\max \left\{x_{1}, \ldots, x_{g}\right\}+1$, and let $v=n_{y}$. Then, for each $i=1, \ldots, g$, we have $\left(0:_{I^{v} N} I_{i}\right)=0$, so that $I_{i}$ is $I^{v} N$-regular. Thus by $1.4\left(\right.$ ii), there exists $c \in \mathbb{N}_{0}^{g}$ such that

$$
\operatorname{Ass}_{R}\left(I^{v} N / I^{a} I^{v} N\right)=\operatorname{Ass}_{R}\left(I^{v} N / I^{c} I^{v} N\right)
$$

for all $a \in \mathbb{N}_{0}^{g}$ with $a \geq c$.

Let $w=\max \{k, c(1)+y, \ldots, c(g)+y\}$. Define $d:=n_{w}$, and let $s, t \in \mathbb{N}^{g}$ with $s \geq t \geq d$. Then there is an exact sequence

$$
0 \longrightarrow I^{t} N / I^{s} N \longrightarrow M / I^{s} N \longrightarrow M / I^{t} N \longrightarrow 0,
$$

and so

$$
\operatorname{Ass}_{R}\left(M / I^{s} N\right) \subseteq \operatorname{Ass}_{R}\left(I^{t} N / I^{s} N\right) \cup \operatorname{Ass}_{R}\left(M / I^{t} N\right) .
$$

Now since $s-v \geq t-v \geq d-v \geq c$,

$$
\begin{aligned}
\operatorname{Ass}_{R}\left(I^{t} N / I^{s} N\right) \subseteq \operatorname{Ass}_{R}\left(I^{v} N / I^{s-v} I^{v} N\right) & =\operatorname{Ass}_{R}\left(I^{v} N / I^{t-v} I^{v} N\right) \\
& \subseteq \operatorname{Ass}_{R}\left(M / I^{t} N\right) .
\end{aligned}
$$

Therefore $\operatorname{Ass}_{R}\left(M / I^{s} N\right) \subseteq \operatorname{Ass}_{R}\left(M / I^{t} N\right)$. Clearly for any $u \in \mathbb{N}^{g}$ with $u \geq d$ there exists $e \in \mathbb{N}$ such that $e d \geq u$, and hence

$$
\operatorname{Ass}_{R}\left(M / I^{d} N\right)=\operatorname{Ass}_{R}\left(M / I^{e d} N\right) \subseteq \operatorname{Ass}_{R}\left(M / I^{u} N\right) \subseteq \operatorname{Ass}_{R}\left(M / I^{d} N\right) .
$$

1.6 Theorem. Let $R$ be a commutative ring (not necessarily Noetherian), let $M$ be a Noetherian $R$-module and let $N$ be a submodule of $M$.

(i) Let $\left(a_{m}\right)_{m \in \mathbb{N}}$ be a sequence in $\mathbb{N}_{0}^{g}$ with $a_{i} \leq a_{i+1}$ for all $i \in \mathbb{N}$. Then $\operatorname{Ass}_{R}\left(M / I^{a_{m}} N\right)$ is ultimately constant for large $m$.

(ii) Furthermore, there exists $d \in \mathbb{N}^{g}$ such that $\operatorname{Ass}_{R}\left(M / I^{n} N\right)$ is independent of $n$ for all $n \geq d$.

Proof. Clearly $M$ has a natural structure as an $R /\left(0:_{R} M\right)$-module with the same submodule structure as the $R$-module, and so is still Noetherian. Let $b \in \mathbb{N}_{0}^{g}$. Since $\left(0:_{R} M\right) \subseteq\left(0:_{R} M / I^{b} N\right), M / I^{b} N$ is a Noetherian $R /\left(0:_{R} M\right)$-module. Let $\phi:$ $R \longrightarrow R /\left(0:_{R} M\right)$ be the natural homomorphism, and $\phi^{*}: \operatorname{Spec}\left(R /\left(0:_{R} M\right)\right) \longrightarrow$ $\operatorname{Spec}(R)$ be the induced map. Then by $[\mathrm{Ru}$, Theorem $5.2(\mathrm{i})]$, we see that

$$
\operatorname{Ass}_{R}\left(M / I^{b} N\right)=\phi^{*}\left(\operatorname{Ass}_{R /\left(0:_{R} M\right)}\left(M / I^{b} N\right)\right) .
$$

Hence since $R /\left(0:_{R} M\right)$ is a Noetherian ring the results follow from 1.5. 


\section{AtTAChed PRIME IDEALS}

2.1 Matlis duality. The classical duality originally developed by Matlis (see [Ma, Section 4]) dealt with a complete Noetherian local ring; it can, in fact, also be applied to a complete Noetherian semi-local ring (see [S-T, 3.5]). We shall be making substantial use of this theory, and so we shall include a summary for the reader. Let $R$ be a complete semi-local Noetherian ring, and let $\mathfrak{m}_{1}, \ldots, \mathfrak{m}_{n}$ be the distinct maximal ideals of $R$. Set $S:=\bigoplus_{j=1}^{n} R / \mathfrak{m}_{j}$ and $E:=E(S)$, the injective envelope of $S$. We shall use $D$ to denote the additive, exact, $R$-linear [N, p.35] functor $\operatorname{Hom}_{R}(-, E)$ from the category of all $R$-modules and $R$-homomorphisms to itself. For each $R$-module $T$, let

$$
\mu_{T}: T \longrightarrow D D(T)=\operatorname{Hom}_{R}\left(\operatorname{Hom}_{R}(T, E), E\right)
$$

be the natural $R$-homomorphism for which $\left(\mu_{T}(x)\right)(f)=f(x)$ for all $x \in T$ and $f \in \operatorname{Hom}_{R}(T, E)$.

Then Matlis duality states (among other things) that, whenever $M$ is a finitely generated $R$-module, $\mu_{M}: M \longrightarrow D D(M)$ is an isomorphism and $D(M)$ is an Artinian $R$-module, and whenever $A$ is an Artinian $R$-module, $\mu_{A}: A \longrightarrow D D(A)$ is an isomorphism and $D(A)$ is a Noetherian $R$-module.

2.2 Lemma. Let the situation be as in 2.1. Let $A$ be an Artinian $R$-module, let $B$ be a submodule of $A$ and let $M:=D(A)$. Then there exists a submodule $N$ of $M$ such that the sets $\operatorname{Ass}_{R}(M / J N)$ and $\operatorname{Att}_{R}\left(B:_{A} J\right)$ are equal for all ideals $J$ of $R$.

Proof. The argument of [S2, Corollary 2.7(ii)] can be extended to this case to show that, whenever $M^{\prime}$ is a Noetherian $R$-module, then $\operatorname{Att}_{R}\left(D\left(M^{\prime}\right)\right)=\operatorname{Ass}_{R}\left(M^{\prime}\right)$.

We know from Matlis duality that there exists a Noetherian $R$-module $L$ and an isomorphism $\psi: D(L) \stackrel{\cong}{\longrightarrow} A / B$. It is easily shown that

$$
0 \longrightarrow\left(0:_{A / B} J\right) \stackrel{\beta}{\longrightarrow} A / B \stackrel{D(i) \circ \psi^{-1}}{\longrightarrow} D(J L) \longrightarrow 0
$$

is an exact sequence, where $\beta$ is the inclusion map and $i$ is the inclusion map of $J L$ into $L$. It follows from this that

$$
0 \longrightarrow J(D(A / B)) \stackrel{\pi}{\longrightarrow} D(A / B) \stackrel{D(\beta)}{\longrightarrow} D\left(0:_{A / B} J\right) \longrightarrow 0
$$

is an exact sequence, where

$$
\pi=\left.\left.D(\psi)^{-1} \circ D D(i) \circ \mu_{J L} \circ \mu_{L}^{-1}\right|_{J(D D(L))} \circ D(\psi)\right|_{J(D(A / B))} .
$$

Hence from the commutative diagrams

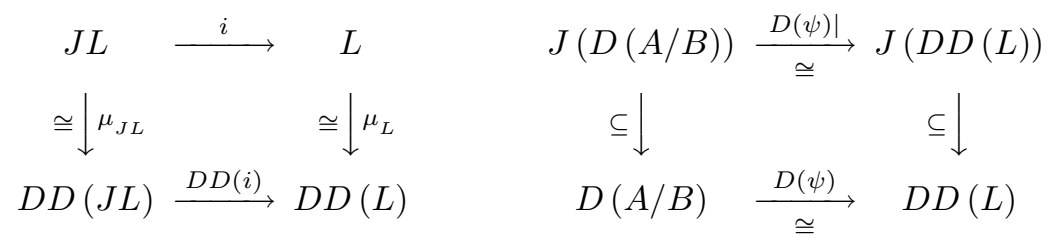


we see that $\pi$ is just inclusion, and so $\operatorname{Ker}(D(\beta))=J(D(A / B))$. Therefore, applying the Snake Lemma to the commutative diagram

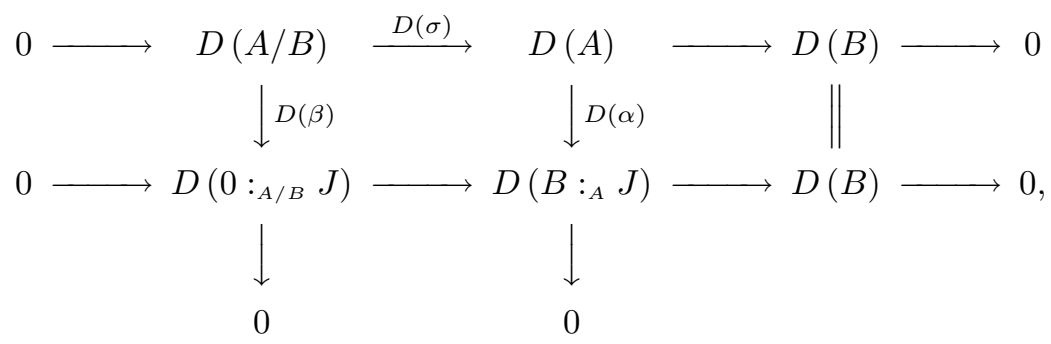

where $\alpha$ is the inclusion map of $\left(B:_{A} J\right)$ into $A$ and $\sigma: A \longrightarrow A / B$ is the canonical epimorphism, we obtain $\operatorname{Ker}(D(\alpha))=J(D(\sigma)(D(A / B)))$.

Hence $D\left(B:_{A} J\right) \cong M / J N$ where $N:=(D(\sigma)(D(A / B)))$ and therefore, since the choice of $N$ is not dependent upon $J$, the proof is finished.

2.3 Theorem. Let $R$ be a complete semi-local Noetherian ring, let $A$ be an Artinian $R$-module and let $B$ be a submodule of $A$.

(i) Let $\left(a_{m}\right)_{m \in \mathbb{N}}$ be a sequence in $\mathbb{N}_{0}^{g}$ with $a_{i} \leq a_{i+1}$ for all $i \in \mathbb{N}$. Then $\operatorname{Att}_{R}\left(B:_{A} I^{a_{m}}\right)$ is ultimately constant for large $m$.

(ii) Furthermore, there exists $d \in \mathbb{N}^{g}$ such that $\operatorname{Att}_{R}\left(B:_{A} I^{n}\right)$ is independent of $n$ for all $n \geq d$.

Proof. This follows from 2.1, 2.2 and 1.6.

2.4 Note. (i) Here we shall outline some results concerning Artinian modules; for further details see [S3, Theorem 3.2]. Let $A$ be a non-zero Artinian module over the commutative ring $R$. There exist only finitely many maximal ideals $\mathfrak{m}$ of $R$ for which $\operatorname{Soc}(A)$ has a submodule isomorphic to $R / \mathfrak{m}$ : let the distinct such maximal ideals be $\mathfrak{m}_{1}, \ldots, \mathfrak{m}_{s}$. Set $J:=\bigcap_{i=1}^{s} \mathfrak{m}_{i}$ and

$$
\hat{R}^{(J)}:={\underset{\varliminf}{n}}_{\lim } R / J^{n},
$$

the $J$-adic completion of $R$. Then $A$ has a natural structure as a module over $\hat{R}^{(J)}$, and the ring $R^{\prime}:=\hat{R}^{(J)} /\left(0:_{\hat{R}^{(J)}} A\right)$ is a complete semi-local commutative Noetherian ring. Also the module $A$ is a faithful Artinian module over $R^{\prime}$; moreover, a subset of $A$ is an $R$-submodule if and only if it is an $R^{\prime}$-submodule.

(ii) Let $\phi: R \longrightarrow R^{\prime}$ be the natural map and let $r \in R$. Then the multiplication by $r$ on $A$ has the same effect as multiplication by $\phi(r)$ on $A$ (see [S3, 2.2]).

2.5 Theorem. Let $R$ be a commutative ring (not necessarily Noetherian), let $A$ be an Artinian $R$-module and let $B$ be a submodule of $A$.

(i) Let $\left(a_{m}\right)_{m \in \mathbb{N}}$ be a sequence in $\mathbb{N}_{0}^{g}$ with $a_{i} \leq a_{i+1}$ for all $i \in \mathbb{N}$. Then $\operatorname{Att}_{R}\left(B:_{A} I^{a_{m}}\right)$ is ultimately constant for large $m$.

(ii) Furthermore, there exists $d \in \mathbb{N}^{g}$ such that $\operatorname{Att}_{R}\left(B:_{A} I^{n}\right)$ is independent of $n$ for all $n \geq d$.

Proof. If $A=0$ the result is clear, and so we may assume that $A \neq 0$. Let the notation be as in 2.4. Let $\phi: R \longrightarrow R^{\prime}$ be the natural map and, for an ideal $K$ of 
$R$, let $K^{e}$ denote the extension of $K$ to $R^{\prime}$ under $\phi$. Let $\phi^{*}: \operatorname{Spec}\left(R^{\prime}\right) \longrightarrow \operatorname{Spec}(R)$ be the induced map. Then, by [Ru, Theorem 5.2(ii)], we see that

$$
\operatorname{Att}_{R}\left(B:_{A} I^{n}\right)=\phi^{*}\left(\operatorname{Att}_{R^{\prime}}\left(B:_{A} I^{n}\right)\right) \quad \text { for all } n \in \mathbb{N}_{0}^{g},
$$

and so it suffices to show that (i) $\operatorname{Att}_{R^{\prime}}\left(\left(B:_{A} I^{a_{m}}\right)\right)$ is ultimately constant for large $m$, and (ii) there exists $d \in \mathbb{N}^{g}$ such that $\operatorname{Att}_{R^{\prime}}\left(B:_{A} I^{n}\right)$ is independent of $n$ for all $n \geq d$. Let $\left(I^{e}\right)^{n}$ denote $\left(I_{1}^{e}\right)^{n(1)} \ldots\left(I_{g}^{e}\right)^{n(g)}$ for all $n \in \mathbb{N}_{0}^{g}$. Then using the natural structure of $A$ as an $R^{\prime}$-module we see from 2.4(ii) that

$$
\left(B:_{A} I^{n}\right)=\left(B:_{A}\left(I^{n}\right)^{e}\right)=\left(B:_{A}\left(I^{e}\right)^{n}\right)
$$

for all $n \in \mathbb{N}_{0}^{g}$. Hence the results follow from 2.4(i) and 2.3.

\section{REFERENCES}

[B] M. Brodmann, Asymptotic stability of Ass $\left(M / I^{n} M\right)$, Proc. Amer. Math. Soc. 74 (1979), 16-18. MR 80c: 13012

[K-M-R] D. Katz, S. McAdam and L. J. Ratliff, Jr., Prime divisors and divisorial ideals, J. Pure Appl. Algebra 59 (1989), 179-186. MR 90g:13030

[Ma] E. Matlis, Injective modules over Noetherian rings, Pacific J. Math. 8 (1958), 511-528. MR 20:5800

[Mc] S. McAdam, Primes associated to an ideal, Contemporary Mathematics, vol. 102, Amer. Math. Soc., Providence, 1989. MR 90m:13004

[N] D. G. Northcott, An introduction to homological algebra, Cambridge Univ. Press, Cambridge, 1960. MR 22:9523

[Ra] L. J. Ratliff, Jr., On prime divisors of $I^{n}$, n large, Michigan Math. J. 23 (1976), 337-352. MR 56:15626

[Ru] D. E. Rush, Asymptotic primes and integral closure in modules, Quart. J. Math. Oxford Ser. (2) 43 (1992), 477-499. MR 93m:13009

[S1] R. Y. Sharp, Asymptotic behaviour of certain sets of attached prime ideals, J. London Math. Soc. (2) 34 (1986), 212-218. MR 87i:13007

[S2] _ A method for the study of Artinian modules, with an application to asymptotic behavior, Commutative algebra (Proceedings of a Microprogram held June 15-July 2, 1987), Mathematical Sciences Research Institute Publications, vol. 15, Springer-Verlag, New York, 1989, pp. 443-465. MR 91a:13011

[S3] _ Artinian modules over commutative rings, Math. Proc. Cambridge Phil. Soc. 111 (1992), 25-33. MR 93a:13009

[S-T] R. Y. Sharp and Y. Tiraş, Asymptotic behaviour of integral closures of ideals relative to Artinian modules, J. Algebra 153 (1992), 262-269. MR 94b:13007

Pure Mathematics Section, School of Mathematics and Statistics, University of Sheffield, Hicks Building, Sheffield, S3 7RH, United Kingdom

E-mail address: a.kingsbury@sheffield.ac.uk

E-mail address: r.y.sharp@sheffield.ac.uk 